\title{
The Crystal and Mesophase Structure of Hexakis(alkylsulfono)- benzene Homologues by X-Ray Diffractometry*
}

\author{
N. Spielberg**, Z. Luz, and R. Poupko \\ Department of Isotope Research, Weizmann Institute of Science, Rehovot 76 100, Isreal \\ K. Praefcke***, B. Kohne, and J. Pickardt \\ Fachbereich Synthetische und Analytische Chemie, Technische Universität Berlin, \\ D-1000 Berlin 12, Germany \\ K. Horn \\ Fritz-Haber-Institut der Max-Planck-Gesellschaft, D-1000 Berlin 33, Germany
}

Z. Naturforsch. 41 a, 855-860 (1986); received February 21, 1986

A powder X-ray diffractometer study of hexakis(tridecylsulfono)benzene (HASB13) has been carried out over the temperature range 20 to above $80^{\circ} \mathrm{C}$. In this range three phase transitions are observed by sharp discontinuities in the diffraction pattern indicating a solid-solid, solidmesophase, and mesophase-liquid transition. The mesomorphic phase is identified as a hexagonal columnar discotic mesophase, $D_{\text {hd }}$, with intercolumnar spacing of $25.7 \AA$ and average stacking distance of $4.9 \AA$. Both distances are independent of temperature but there appears to be a gradual increase in the stacking disorder as the temperature is increased. The magnitude of the intercolumnar distance suggests a considerable degree of side chain disorder. A detailed X-ray diffraction study was also performed at room temperature on a single crystal of hexakis(propylsulfono)benzene (HASB3), which is not mesogenic. The results provide detailed information on the structure of HASB 3 which is used in the interpretation of HASB 13 results.

\section{Introduction}

The "classical" discotic liquid crystalline compounds consist of a central rigid core to which several flexible alkyl side chains are attached by means of ethers or ester bonds [1,2]. More recently discotic compounds were discovered with different linking groups which also exhibit such mesophases [3-5]. A particular example is the homologous series hexakis(alkylsulfono)benezene (HASB) in which the bridging is via $\mathrm{SO}_{2}$ groups $[3,5]$. Homologues of this series with $n=7$ to 15 carbons per side chain exhibit a stable mesophase in the temperature range 40 to $140^{\circ} \mathrm{C}$ (see Figure 1) [5]. On the basis of differential scanning calorimetry,

Reprint requests to Prof. K. Praefcke, Fachbereich Synthetische und Analytische Chemie der Technischen Universität Berlin, D-1000 Berlin 12, Germany.

* Part XXXI on liquid crystalline and part LXXI on organic sulfur compounds, respectively. Part XXX and part LXX, respectively: B. Kohne, K. Praefcke, and W. Stephan, Chimia 40, 14 (1986).

** Erna and Jakob Michael Visiting Professor, 1983/84. Permanent address: Department of Physics, Kent State University, Kent, Ohio 44 242, USA.

*** Minerva Fellow, Weizmann Institute of Science, 1985/86. optical microscopy and nuclear magnetic resonance spectroscopy this mesophase was tentatively identified as a uniaxial columnar mesophase with negative anisotropic magnetic susceptibility $(\Delta \chi<0)$ [5]. In the present paper we report a detailed X-ray powder diffraction study of the $n=13$ member of the series (HASB13). The results are consistent with the previous study and provide additional quantitative information on the structure of the mesophase, in particular with regard to the intra- and intercolumnar spacing and the overall symmetry of the mesophase.

To obtain structural information on the sulfonobenzene core we also performed a detailed structural study on a single crystal of HASB3 which is not mesogenic. This study provided detailed atomic coordinates on the HASB3 molecules which are used in the discussion of the mesophase structure of the higher homologues.

\section{Experimental}

The powder X-ray measurements on the HASB13 homologue were made using a modified vertical

0340-4811 / $86 / 0600-0855 \$ 01.30 / 0$. - Please order a reprint rather than making your own copy. 


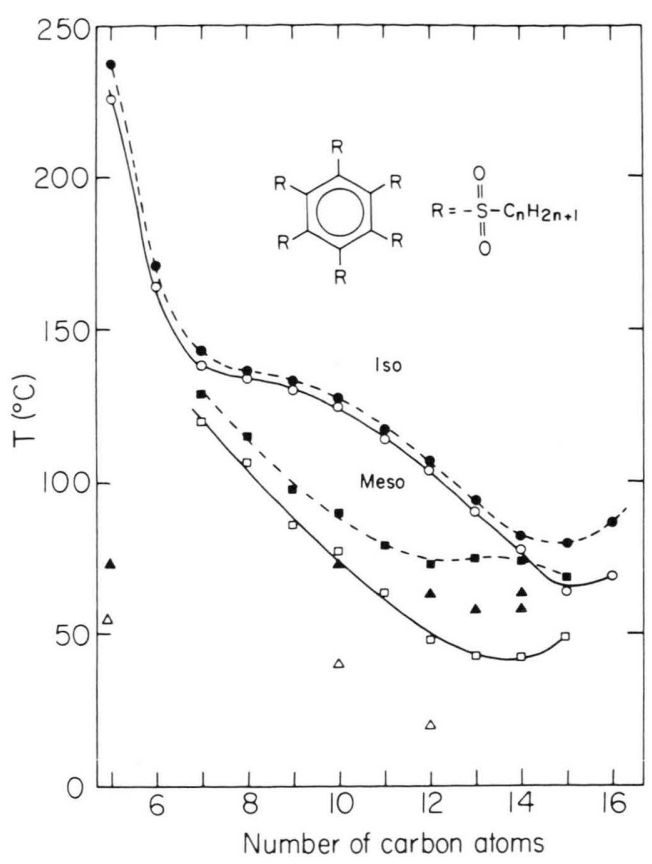

Fig. 1. Transition temperatures in the hexakis(alkylsulfono)benzene series for $5 \leqq n \leqq 16$, based on DSC measurements [5]. Open symbols are for transitions with decreasing temperature; solid symbols for increasing temperature. Circles are for the isotropic-mesophase, squares for the mesophase-solid, and triangles for the solid-solid transition.

scanning Philips diffractometer with transmission specimen and focussing LiF (200) monochromator adjusted for $\mathrm{Cu} \mathrm{K} \alpha$ radiation (weighted wavelength $1.5418 \AA$ ) in the incident beam, and a temperature controlled specimen stage [6]. Intensities were recorded with a $\mathrm{NaI}(\mathrm{Tl})$ scintillation detector and were transferred to a central computer for further processing. Some additional measurements were made using a small angle scattering apparatus having a two-dimensional position sensitive detector [7].

Samples of HASB13 for the powder diffraction measurements were hermetically sealed in discshaped specimens which were $0.5 \mathrm{~mm}$ thick and covered at top and bottom with $25 \mu$ aluminum foils. The previous work [5] indicated that it is possible, by slowly cooling a bulk sample from the isotropic phase in a strong magnetic field, to prepare specimens having a two-dimensional distribution of domains with directors lying in a plane perpendicular to the direction of the field. However, attempts to align the samples in the above specimens were unsuccessful, apparently due to strong wall effects. Consequently all results on HASB13 refer to unaligned "powder" samples.

The single crystal measurements on HASB3 were made at room temperature using a Syntex $\mathrm{P} 2_{1}$ diffractometer equipped with a graphite monochromatized $\operatorname{MoK} \alpha$ radiation source (weighted wavelength $0.7107 \AA$ ) [8]. The single crystal dimensions were $0.3 \times 0.35 \times 0.5 \mathrm{~mm}$. Of the 3167 independent reflections, 2770 were used for the structure refinement. The data were corrected for dispersion, and anisotropic temperature factors were determined. The $R$ factor for the sructure was 0.0477 .

The compounds were synthesized as previously described $[3,5]$.

\section{Results and Discussion}

Diffraction patterns obtained while cooling a sample of HASB13 from the isotropic phase exhibited characteristic features of an amorphous liquid, hexagonal mesophase and two solid phases. The transition temperatures were $\left(\right.$ in ${ }^{\circ} \mathrm{C}$ )

$$
\mathrm{S}_{2} \stackrel{44 \pm 4}{\longleftarrow} \mathrm{S}_{1} \stackrel{63 \pm 1}{\longleftarrow} \mathrm{M} \stackrel{80 \pm 1}{\longleftarrow} \mathrm{I} .
$$

These results are in reasonable agreement with those previously reported [5], however, exact comparison is not possible since different techniques gave somewhat different transition temperatures, presumably due to hysteresis and supercooling effects which may depend on the sizes and shapes of the samples.

\section{The Solid Phases}

Before discussing the diffraction data of the mesophase we briefly comment on the result obtained from the solid phases. For HASB13 only powder diffraction measurements were made, where at least 14 well resolved peaks were observed. Figures 2 and 3 summarize the d-spacings and relative intensities of most of these peaks as functions of temperature, while Fig. 4 contains intensity data of three diffraction peaks which are not included in Figure 3. The latter peaks exhibit a clear discontinuity in the diffracted intensity in the temperature range 40 to $48^{\circ} \mathrm{C}$. We believe that this discontinuity is associated with a solid-solid transi- 


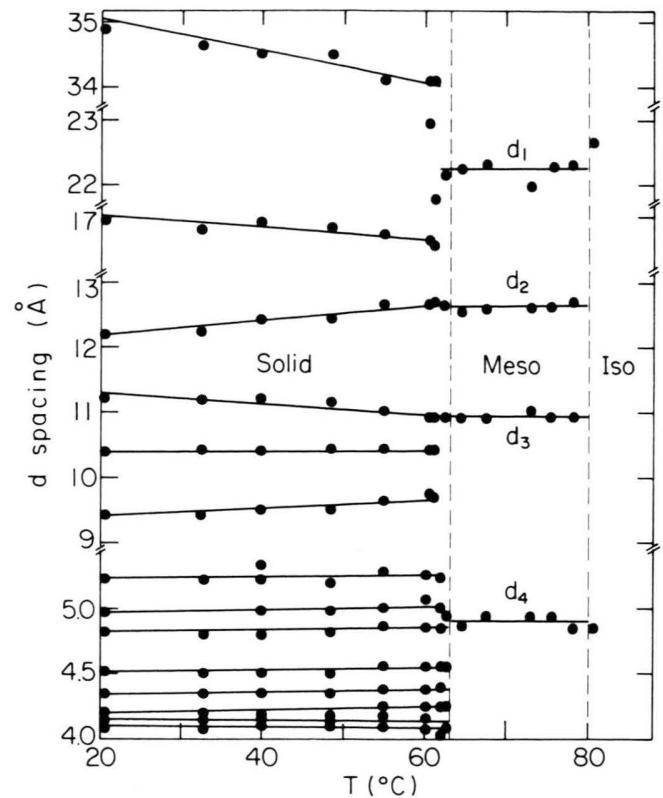

Fig. 2. d-Spacings as functions of temperature obtained from the X-ray diffraction of powder HASB13. Note that the scale for the 4 to $5.3 \AA$ range is twice as large as for the other ranges.

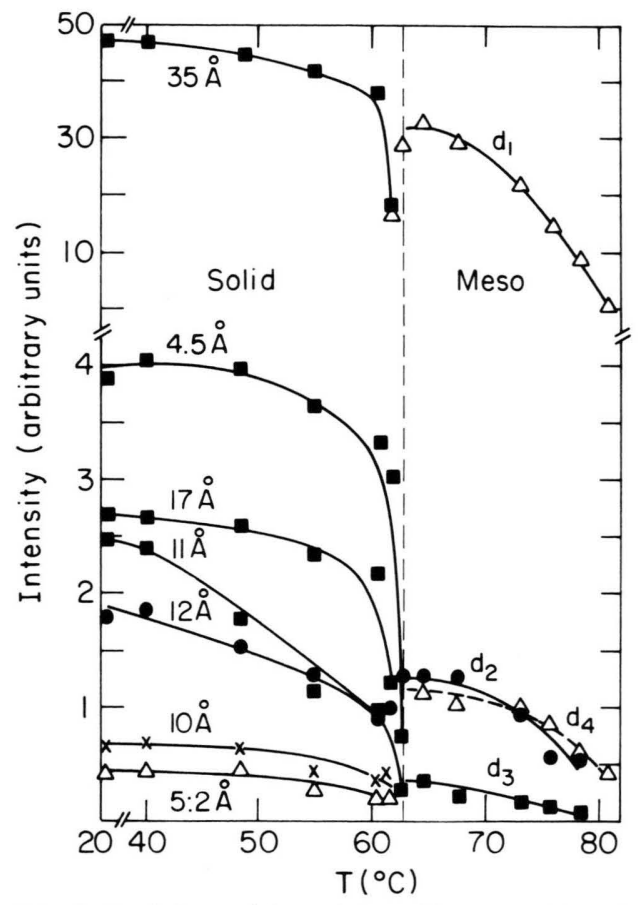

Fig. 3. Peak intensities of the diffraction lines in HASB13 as functions of temperature. Note the break on the temperature axis between 20 and $40{ }^{\circ} \mathrm{C}$, and the two different scales on the intensity axis. The lines are labeled according to their $d$ values at $20^{\circ} \mathrm{C}$, for the solid phase, and $73^{\circ} \mathrm{C}$ for the mesophase (see Figure 2).

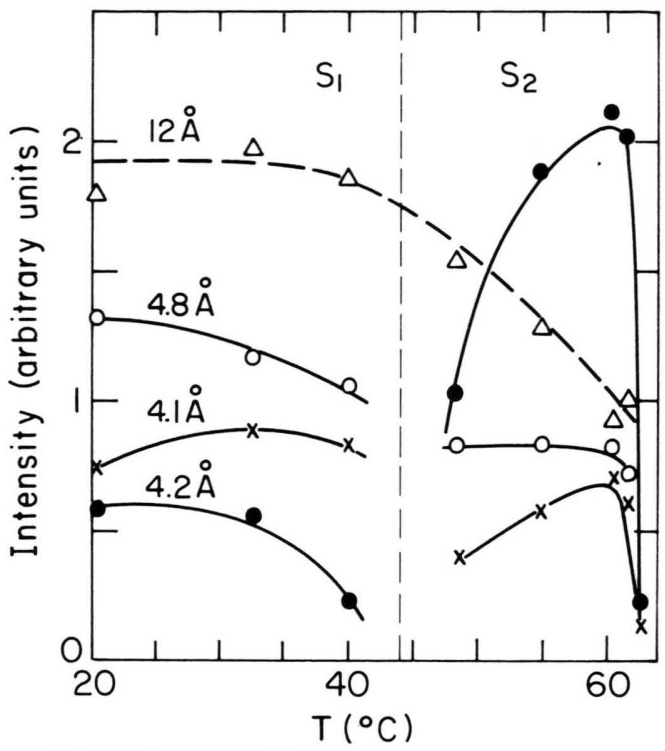

Fig. 4. Peak intensities of three "high angle" lines of HASB13 (not shown in Fig. 3) a functions of temperature within the solid range. These lines are plotted on an expanded scale to emphasize the discontinuity in the scattering intensity at the solid-solid transition. For comparison the intensity of the $12 \AA$ peak from Fig. 3, which shows no such discontinuity is also drawn (dashed line). The lines are labeled according to their $d$ values at $20^{\circ} \mathrm{C}$.

Table 1. Unit cell parameters of solid hexakis(propylsulfono) benzene.

\begin{tabular}{|c|c|c|c|}
\hline & s $(\AA)$ & & (degrees) \\
\hline$a$ & 13.844 & $\alpha$ & 95.88 \\
\hline$b$ & 14.538 & $\beta$ & 109.44 \\
\hline$c$ & 10.215 & $\gamma$ & 114.19 \\
\hline
\end{tabular}

Volume $=1699(\AA)^{3}$. Density $($ calcuated $)=1.44 \mathrm{gm} / \mathrm{cm}^{3}$.

tion which was previously observed by differential scanning calorimetry (although only upon heating and at a somewhat higher temperature) [5]. Since the diffraction patterns correspond to a powder sample it is not possible to draw definitive conclusions regarding the structure of the solid phases. The results rule out however hexagonal or higher symmetries.

$\mathrm{X}$-ray measurements were also performed on a single crystal of the homologue HASB3. The details of the raw data have been submitted to the Fachinformationszentrum [9]. The crystal is triclinic, belonging to the PI space group and consisting of two molecules per unit cell. The unit cell parameters are given in Tab. 1, and the molecular geometry is 


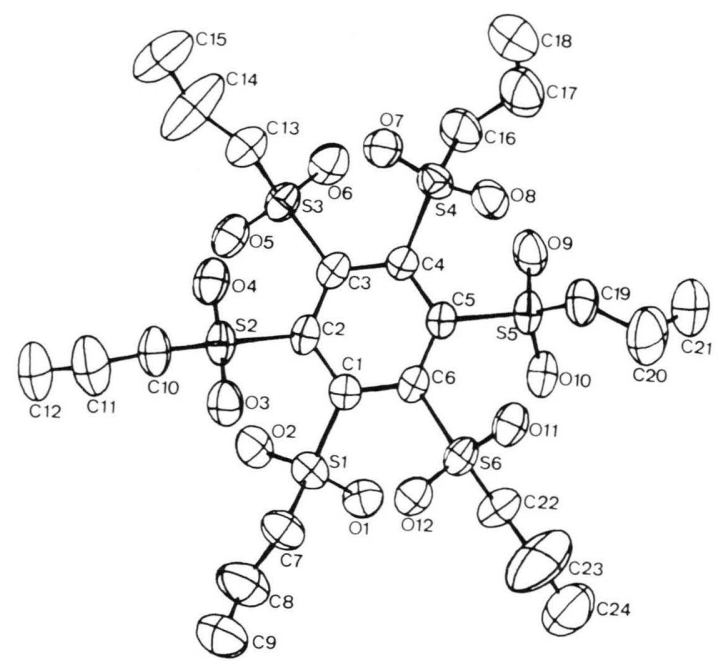

Fig. 5. the molecular structure of HASB3 from the single crystal X-ray diffraction data.

shown in Figure 5. The benzene ring is planar and the chains appear to be fully stretched. The displacements of the side chain atoms from the plane of the benzene ring are given in. Table 2, and average interatomic distances and bond angles are summarized in Table 3. It may be seen that the oxygen atoms of successive $\mathrm{SO}_{2}$ groups are alternately oriented above and below the benzene plane [10]. This rules out the possibility of an alternative structure in which the six sulfurs are bonded to each other via oxygen bridges coplanar with the benzene ring to form a macroheterocyclic ring surrounding the benzene core. Such a structure is also ruled out by ESCA measurements which indicated that the compound has just one type of oxygen rather than two, as would be expected for the alternative multisulfurane-oxide structure [11].

\section{The Mesophase}

As the temperature of a HASB13 sample is raised to above $63^{\circ} \mathrm{C}$ there is a sharp discontinuous change in the diffraction pattern, which corresponds to the solid-mesophase transition. In the mesophase only four diffraction peaks are observed, three of which of quite low relative intensity. The temperature dependence of the d-spacing and relative intensities are shown in Figs. 2 and 3. The spacings appear to be quite constant over the whole stability range of the mesophase $\left(63\right.$ to $\left.80^{\circ} \mathrm{C}\right)$ while the
Table 2. Distances (in $\AA$ ) of side chain atoms from plane of benzene ring in solid hexakis(propylsulfono)benzene. (Numbering as in Fig. 5.)

\begin{tabular}{lclc}
\hline Atom & Distance & Atom & Distance \\
\hline S1 & 0.75 & O 1 & 1.55 \\
S2 & -0.77 & O2 & 1.48 \\
S3 & 0.69 & O3 & -1.51 \\
S 4 & -0.58 & O4 & -1.54 \\
S5 & 0.555 & O5 & 1.36 \\
S6 & -0.63 & O6 & 1.49 \\
C7 & -0.46 & O7 & -1.29 \\
C10 & 0.42 & O8 & -1.36 \\
C 13 & -0.61 & O9 & 1.34 \\
C16 & 0.82 & O 10 & 1.26 \\
C19 & -0.86 & O 11 & -1.46 \\
C 22 & 0.66 & O 12 & -1.32 \\
\hline
\end{tabular}

Table 3. Average bond lengths and bond angles in solid hexakis (propylsulfono) benzene.

\begin{tabular}{|c|c|c|c|c|}
\hline & & $\begin{array}{l}\text { Bond length } \\
(\AA)\end{array}$ & & $\begin{array}{l}\text { Bond angle } \\
\text { (degrees) }\end{array}$ \\
\hline \multirow[t]{2}{*}{$\begin{array}{l}\text { Ring: } \\
\text { Chain: }\end{array}$} & $\begin{array}{l}\mathrm{C}-\mathrm{C} \\
\mathrm{C}-\mathrm{S}\end{array}$ & $\begin{array}{l}1.42 \pm 0.01 \\
1.88 \pm 0.003\end{array}$ & $\begin{array}{l}\mathrm{C}-\mathrm{C}-\mathrm{C} \\
\mathrm{C}-\mathrm{S}-\mathrm{C}\end{array}$ & $\begin{array}{l}120 \pm 0.4 \\
115 \pm 2\end{array}$ \\
\hline & $\begin{array}{l}\mathrm{S}-\mathrm{O} \\
\mathrm{S}-\mathrm{C} \\
\mathrm{C}-\mathrm{C}\end{array}$ & $\begin{array}{l}1.46 \pm 0.003 \\
1.82 \pm 0.01 \\
1.52 \pm 0.015\end{array}$ & $\begin{array}{l}\mathrm{O}-\mathrm{S}-\mathrm{O} \\
\mathrm{S}-\mathrm{C}-\mathrm{C} \\
\mathrm{C}-\mathrm{C}-\mathrm{C}\end{array}$ & $\begin{array}{l}117 \pm 0.2 \\
110 \pm 1 \\
113 \pm 3\end{array}$ \\
\hline
\end{tabular}

intensities decrease monotonically as the clearing point is approached. The spacings corresponding to the $d_{1}, d_{2}$, and $d_{3}$ peaks in Fig. 2 are in the ratio $1: 1 / \sqrt{3}: 1 / 2$, suggesting a hexagonal symmetry, in agreement with the NMR results which indicated a uniaxial symmetry for this mesophase [5]. In Table 4 the spacings of these peaks at different temperatures within the mesophase are summarized and compared with the expected spacings for hexagonal symmetry. The agreement is quite satisfactory, yielding an average lattice repeat unit of $a=22.26$ / $\sin 60^{\circ}=25.7 \AA$. By analogy with other discotics we identify this parameter with the average distance between columns in an hexagonal array. The fourth scattering peak at $d=4.9 \AA$ must therefore correspond to the average stacking distance of the molecules with the columns.

By comparison of the single crystal data for HASB3 with those for HASB13 some conclusions as to the conformation of the alkyl side chains in the mesomorphic phase can be drawn. The "molecular diameter" of HASB3, as calculated from the single crystal data is $15.8 \AA$, while the largest d-spacing 


\begin{tabular}{|c|c|c|c|c|c|c|c|}
\hline \multirow{3}{*}{$\begin{array}{l}\text { Temp. } \\
{ }^{\circ} \mathrm{C}\end{array}$} & \multicolumn{5}{|c|}{ d-Spacings $(\AA)$} & \multirow{2}{*}{\multicolumn{2}{|c|}{$\begin{array}{c}\text { Deviations }(\AA) \\
\text { (Meas.-Calc.) }\end{array}$}} \\
\hline & \multicolumn{3}{|c|}{ Measured } & \multicolumn{2}{|c|}{ Calculated } & & \\
\hline & $d_{1}$ & $d_{2}$ & $d_{3}$ & $\sqrt{3} d_{2}$ & $2 d_{3}$ & $\left(d_{1}-\sqrt{3} d_{2}\right)$ & $\left(d_{1}-2 d_{3}\right)$ \\
\hline 60.6 & 22.95 & 12.68 & 10.92 & 21.96 & 21.84 & 0.99 & 1.11 \\
\hline 61.2 & 21.76 & 12.70 & 10.97 & 22.00 & 21.94 & -0.24 & -0.18 \\
\hline 62.5 & 22.14 & 12.65 & 10.92 & 21.91 & 21.84 & 0.23 & 0.30 \\
\hline 64.5 & 22.26 & 12.56 & 10.93 & 21.75 & 21.86 & 0.51 & 0.40 \\
\hline 67.6 & 22.31 & 12.59 & 10.92 & 21.81 & 21.84 & 0.50 & 0.47 \\
\hline 73.0 & 21.98 & 12.61 & 11.02 & 21.84 & 22.04 & 0.14 & -0.06 \\
\hline 75.7 & 22.26 & 12.65 & 10.92 & 21.88 & 21.84 & 0.38 & 0.42 \\
\hline 78.2 & 22.31 & 12.72 & 10.94 & 22.03 & 21.88 & 0.28 & 0.43 \\
\hline \multirow[t]{3}{*}{80.7} & $\begin{array}{r}22.25 \\
\pm 0.34\end{array}$ & $\begin{array}{r}12.64 \\
\pm 0.06\end{array}$ & $\begin{array}{r}10.94 \\
\pm 0.04\end{array}$ & $\begin{array}{r}21.89 \\
\pm 0.10\end{array}$ & $\begin{array}{r}21.88 \\
\pm 0.08\end{array}$ & 0.36 & 0.37 \\
\hline & \multirow{2}{*}{\multicolumn{5}{|c|}{$\begin{array}{l}\text { rms average of all } \delta \text { values } \\
\text { rms average, omitting first two }\end{array}$}} & 0.48 & 0.43 \\
\hline & & & & & & 0.37 & 0.37 \\
\hline
\end{tabular}

Table 4.

Comparison of the experimental and calculated spacings of the low angle diffraction lines in the mesophase of hexakis (tridecylsulfono)benzene. that could be measured in HASB3 is $12.7 \AA$. For HASB13 the diameter of an all trans conformation can be calculated from the result for HASB3 by adding to each side chain a length corresponding to a fully stretched chain of ten carbon atoms. This gives (taking $1.5 \AA$ for the $\mathrm{C}-\mathrm{C}$ bond length and $109.47^{\circ}$ for the $\mathrm{C}-\mathrm{C}-\mathrm{C}$ angle) $40.2 \AA$, which should be compared with a largest d-spacing of $35 \AA$ in solid HASB13. The ratio of these two lengths is very similar to the corresponding figure in HASB3, suggesting that the HASB13 molecules in the solid are also in the all-trans configuration. On going to the mesophase there is a sharp reduction in the largest $\mathrm{d}$-spacing corresponding to a repeat unit of $26 \AA$, indicating that in this phase the side chains are considerably disordered due to the presence of gauche conformations in the side chain.

The measured value of the repeat unit in the mesophase of HASB13 may be compared with corresponding values in other discotic mesophases. Thus for truxenehexadecanoate [12], the repeat unit is $31 \AA$, i.e. about $5 \AA$ larger than in HASB13. The fact that the diameter of the truxene core is larger than that of sulfone derivatives suggests that in both discotic series the side chains are highly disordered. This was also manifest by deuterium NMR studies [13, 14]. It should be noted that while for truxene the repeat units are temperature dependent, for HASB13 they are not.

We have attributed the $4.9 \AA \mathrm{d}$-spacing (peak $d_{4}$ in Figs. 2 and 3 ) to the average distance between molecules within the columns. However since we were unable to align the sample we could not prove that this scattering feature is indeed perpendicular to

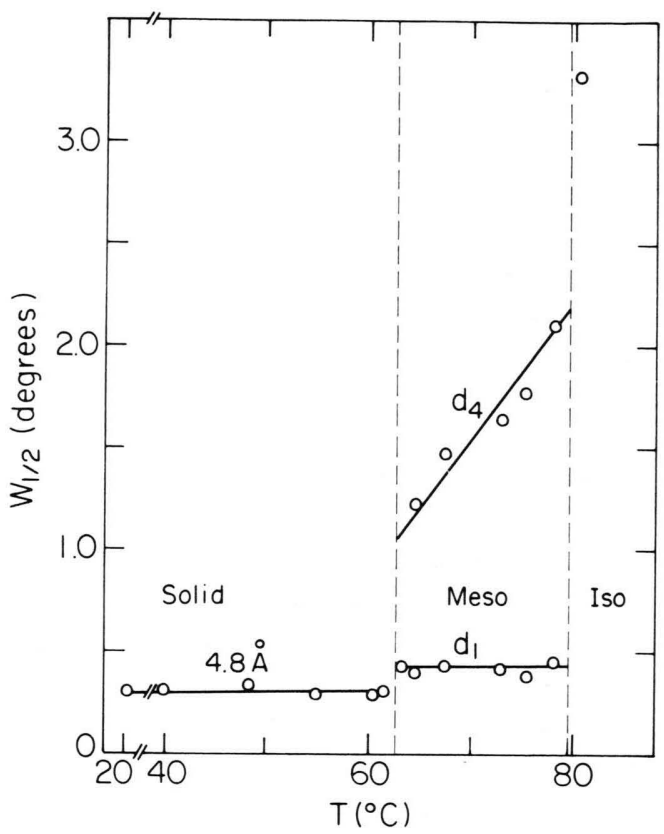

Fig. 6. Width at half-maximum intensity, $W_{1 / 2}$, of X-ray diffraction peaks in the solid and mesophase of HASB13. The $4.8 \AA$ peak is typical of all solid diffractions. At $4.9 \AA$, a width of $1^{\circ} 2 \theta$ corresponds to a range of d-spacings of $\cong 0.3 \AA$.

those attributed to the intercolumnar spacings. The spacing of $4.9 \AA$ is in agreement with that expected from the molecular dimensions of the hexasulfonobenzene core as determined in the solid phase of the HASB3 homologue. As shown in Table 2, the $\mathrm{SO}_{2}$ oxygens are located between 1.3 and $1.5 \AA$ above and below the plane of the benzene core. Addition of the diameter of an oxygen atom gives a distance 
of closest stacking of about $4.2 \AA$, which is significantly lower than $4.9 \AA$, indicating disorder within the column. This disorder is also manifested in the width of the $4.9 \AA$ scattering peak. In Fig. 6 the widths of this peak $\left(d_{4}\right)$ and of the $d_{1}$ peak in the mesophase region are plotted vs. temperature. For the sake of comparison the width of one of the solid peaks is also plotted. It is the $4.8 \AA$ peak, but its behaviour is similar to that of the other solid diffractions. It may be seen that the width of $d_{4}$ is considerably broader than the solid and the other mesophase diffractions, confirming the disordered nature of the columns and identifying the phase as a $D_{\text {hd }}$ columnar discotic. Figure 6 indicates a continuous increase in the disorder until the mesophase transforms to the isotropic liquid.

In the isotropic phase, close to the clearing point two diffraction peaks are still discernible, i.e. at $4.9 \AA$ and at $22.7 \AA$. They are quite weak and very broad. The use of Bragg's equations for their inter-

[1] J. Billard, in: Liquid Crystals of One and Two Dimensional Order (W. Helfrich and G. Heppke, eds.). Springer-Verlag, Berlin 1980, p. 383 . - J. C. Dubois and J. Billard, in: Liquid Crystals and Ordered Fluids, Vol. 4 (A. C. Griffin and J. F. Johnson, eds.). Plenum Press, New York 1984, p. 1043.

[2] D. Goldfarb, Z. Luz, and H. Zimmermann, Israel J. Chem. 23, 341 (1983). - Z. Luz, D. Goldfarb, and H. Zimmermann, in: Nuclear Magnetic Resonance of Liquid Crystals (J. W. Emsley, ed.). D. Reidel Pub. Co. Dordrecht, Holland 1985, p. 343.

[3] W. Poules and K. Praefcke, Chemiker-Ztg. 107, 310 (1983); 107, 374 (1983).

[4] B. Kohne, W. Poules, and K. Praefcke, Chemiker-Ztg. 108, 113 (1984).

[5] K. Praefcke, W. Poules, B. Scheuble, R. Poupko, and Z. Luz, Z. Naturforsch. 39 b, 950 (1984).

[6] D. Goldfarb, Z. Luz, N. Spielberg, and H. Zimmermann, Mol. Cryst. Liq. Cryst. 126, 225 (1985).

[7] K. O. Greulich, J. Ausio, D. Seger, E. Wachtel, and H. Eisenberg, to be published.

[8] $\omega-2 \theta$ scan technique, $2 \theta \leqq 40^{\circ}$, variable scan speed, two standards were measured after each 50 reflections. pretation is of course questionable but the numerical values of the d-spacings suggest that they may correspond to the stacking and intercolumnar distances of clusters or short stacks formed in the isotropic phase as the transition to the mesophase is approached.

\section{Acknowledgements}

This work was partly supported by the Israel Academy of Sciences. We are grateful to E. Wachtel, D. Rabinovitz, J. Halfon, and J. Gorodetzki for advice and help in the X-ray measurements. K.P. acknowledges financial support by the Deutsche Forschungsgemeinschaft ( $\operatorname{Pr} 116 / 8-1,2)$, the Fonds der Chemischen Industrie and the Technische Universität Berlin (Kanzler and PA 1, partnership agreement for scientific collaboration between the Technische Universität Berlin and the Weizmann Institute of Science: Professors Z. Luz and K. Praefcke).

[9] Further details concerning the X-ray analysis and basic data may be obtained from Fachinformationszentrum Energie, Physik, Mathematik, D-7514 Eggenstein-Leopoldshafen (Germany), by specifying the registry number CSD 51920, the authors, and the source.

[10] B. Kohne and K. Praefcke, Chemiker-Ztg. 109, 121 (1985).

[11] B. Kohne, K. Praefcke, K. Horn, and S. Krause, unpublished results; publication in preparation. One of us (K.P.) thanks Professor J. C. Martin, University of Illinois, Urbana-Champaign, School of Chemical Science, Illinois 61801, USA, for a probe of an authentic sulfurane oxide [3,3,3',3'-tetrakis(trifluoromethyl)-1, $1^{\prime}$ spiro-bi[3 H-2,1-benzoxathiole]-1-oxide, see R. S. Michalak and J. C. Martin, J. Amer. Chem. Soc. 102, 5921 (1980)] for comparing ESCA studies.

[12] A. M. Levelut. J. Chim. Phys. 80, 149 (1983).

[13] D. Goldfarb, Z. Luz, and H. Zimmermann, J. Chem. Phys. 78, 7065 (1983).

[14] D. Goldfarb, E. Lifshitz, H. Zimmermann, and Z. Luz, J. Chem. Phys. 82, 5155 (1985). 